\title{
The role of interstitial brachytherapy in the management of primary radiation therapy for uterine cervical cancer
}

\author{
Naoya Murakami, MD, PhD!, Kazuma Kobayashi, MD!, Tomoyasu Kato, MD, PhD², Satoshi Nakamura, MSc', \\ Akihisa Wakita, MSc', Hiroyuki Okamoto, PhD', Satoshi Shima, MD', Keisuke Tsuchida, MD', Tairo Kashihara, MD! \\ Ken Harada, MD, PhD!, Kana Takahashi, MD!, Rei Umezawa, MD, PhD!, Koji Inaba, MD, PhD!', Yoshinori Ito, MD! \\ Hiroshi lgaki, MD, PhD', Jun Itami, MD, PhD' \\ 'Department of Radiation Oncology, 2Department of Gynecologic Oncology, National Cancer Center Hospital, Chuo-ku, Tokyo, Japan
}

\begin{abstract}
Purpose: The aim of this study was to report the clinical results of uterine cervical cancer patients treated by primary radiation therapy including brachytherapy, and investigate the role of interstitial brachytherapy (ISBT).

Material and methods: All consecutive uterine cervical cancer patients who were treated by primary radiation therapy were reviewed, and those who were treated by ISBT were further investigated for clinical outcomes and related toxicities.

Results: From December 2008 to October 2014, 209 consecutive uterine cervical cancer patients were treated with primary radiation therapy. Among them, 142 and 42 patients were treated by intracavitary and hybrid brachytherapy, respectively. Twenty-five patients $(12 \%)$ were treated by high-dose-rate (HDR)-ISBT. Five patients with distant metastasis other than para-aortic lymph node were excluded, and 20 patients consisted of the analysis. Three-year overall survival (OS), progression-free survival (PFS), and local control (LC) rate were $44.4 \%, 38.9 \%$, and $87.8 \%$, respectively. Distant metastasis was the most frequent site of first relapse after HDR-ISBT. One and four patients experienced grade 3 and 2 rectal bleeding, one grade 2 cystitis, and two grade 2 vaginal ulcer.

Conclusions: Feasibility and favorable local control of interstitial brachytherapy for locally advanced cervical cancer was demonstrated through a single institutional experience with a small number of patients.

J Contemp Brachytherapy 2016; 8, 5: 391-398 DOI: $10.5114 /$ jcb.20l6.62938
\end{abstract}

Key words: cervical cancer, primary radiotherapy, interstitial brachytherapy.

\section{Purpose}

In the management of cervical cancer with definitive radiation therapy, brachytherapy plays a central role because it has intrinsic physical property of steep dose gradient and has a potential to deliver relatively large dose while sparing the surrounding organ at risk (OAR) as low as possible [1,2]. Small tumors, which are within isodose line generated by conventional intracavitary brachytherapy (ICBT), consisted of intrauterine (tandem) and vaginal (ovoid/ring) sources, calculated based on the traditional Manchester principles [3,4], can be well controlled. Tumors, which are slightly larger than isodose volume created by ICBT, can be well treated by image-guided adaptive brachytherapy (IGABT) $[5,6,7,8]$ with or without additional interstitial needle insertion (hybrid brachytherapy - HBT) $[9,10,11,12]$. On the other hand, guidelines from the American Brachytherapy Society recommends high-dose-rate (HDR) interstitial brachytherapy (ISBT) for cervical cancer patients in certain clinical situations such as bulky lesions, a narrow vaginal apex, inability to enter the cervical os, extension to the lateral parametria or pelvic sidewall, and lower vaginal extension [1]. In ISBT, multiple applicators insertion is required and it is more invasive compared to ICBT or HBT technique with patients laid on bed overnight with the applicators in place during treatment period. However, a certain groups of patients exists whose tumors are too large to be well covered by ICBT or HBT.

In this study, consecutive uterine cervical patients in our department treated by primary radiation therapy including brachytherapy were overviewed and the role of ISBT was explored.

\section{Material and methods}

Principles of management for locally advanced uterine cervical cancer in our institution were described in our previous report [13]. If patients were under 75 years old 
and had adequate organ function, concurrent chemoradiation therapy with weekly cisplatin $\left(40 \mathrm{mg} / \mathrm{m}^{2} /\right.$ week $)$ was delivered. External beam radiation therapy (EBRT) was delivered by 3D conformal 4 -fields box technique with 15 MV linear accelerator (Clinac iX, Varian Medical System, Palo Alto, CA, USA). The common EBRT fields included whole uterus, parametrium, upper part of vagina down to the level of lower border of the obturator foramens, and the draining pelvic lymph nodes up to the level of the common iliac (L4/5 junction). The subclinical regional lymph node clinical target volume (CTV) was contoured according to the guideline [14]. If the primary lesion involved lower third of vagina or there were clinically palpable metastatic inguinal lymph nodes, inguinal regions were also included in EBRT portals. During radiation therapy, tumor response was assessed weekly by an attending radiation oncologist through gynecological examination. To lower the rectum and the bladder dose, $4 \mathrm{~cm}$-width of central shield (CS) was inserted after 30$40 \mathrm{~Gy}$ of whole pelvic radiation therapy (WPRT). The initiation of CS depends upon the tumor shrinkage according to Japan Society of Gynecologic Oncology guidelines [15]. In cases with poor tumor response against EBRT, no CS was utilized. Total pelvic sidewall dose was $50 \mathrm{~Gy}$ in 25 fractions. After the CS was initiated, HDR-ISBT was delivered. All brachytherapy was carried out by ${ }^{192} \mathrm{Ir}$ remote after loading system (RALS, Microselectron HDR ${ }^{\mathrm{TM}}$, Nucletron, Veennendaal, The Netherlands). From 2008, computed tomography (CT)-based image-guided ICBT for uterine cervical cancer patients was started in our department [16], and CT-based image-guided HDR-ISBT was started in the same year. Hybrid brachytherapy has started from 2010 for tumors with mild to moderate parametrial invasion that could not be adequately covered with ICBT $[16,17]$. Indication for application of ISBT was according to ABS guideline as mentioned above [1].

The detailed procedure of HDR-ISBT for patients with cervical cancer was described in our previous studies $[18,19,20]$. In brief, transperineal needle applicators were inserted under either the combination of general and epidural, or the combination of local anesthesia and sedation with the patients in lithotomy position. Transperineal needle insertion was guided by transrectal ultrasound (TRUS). Whenever possible, a tandem was also inserted under TRUS guidance [21]. Simulation CT was carried out by large-bore CT (Aquilion ${ }^{\circledR}$ LG, Toshiba, Tokyo, Japan), which could take images with patients lying in the lithotomy position with applicators in place without moving patients. The image-guided brachytherapy planning was performed based on those CT images with slice interval of $2 \mathrm{~mm}$. Treatment planning was done with a brachytherapy planning system (Oncentra ${ }^{\circledR}$ Nucletron, Veenendaal, The Netherlands). The SyedNeblett perineal template (Best Medical International, Inc., Springfield, VA, USA) was used because it has an advantage of ensuring parallelism and stability of applicator needles throughout the treatment period. A few titanium seed markers were inserted into the target and these markers were used as a reference for daily correction of needle deviation. The CTV was defined based on the CT image taken after needle insertion with patients in the lithotomy position. Information obtained from gynecological examination performed immediately before needle insertion, intra-operative TRUS images, and the most recent magnetic resonance images (MRIs), which were taken in majority of cases, were also taken into consideration for CTV determination. Reference points were set on the surface of the CTV and the prescribed dose was delivered to those points. Dose calculation was performed by geometrical optimization or dose point volume optimization, followed by manual graphical modification to cover the CTV with the prescription dose while keeping the dose to OAR to a minimum. Hyper-dose sleeve, which represents $200 \%$ of prescribed dose of a certain applicator should not fuse to that of another applicator in order not to increase the high dose volume. The dose constraint of combining dose of WPRT and HDR-ISBT for the rectum and the bladder $\mathrm{D}_{2 \mathrm{cc}}$ was $75 \mathrm{~Gy}$ and $90 \mathrm{~Gy}$ $\left(\mathrm{EQD}_{2}, \alpha / \beta=3 \mathrm{~Gy}\right)$, respectively [6]. Our previous study found that vaginal $\mathrm{D}_{2 \mathrm{cc}}$ greater than $145 \mathrm{~Gy}\left(\mathrm{EQD}_{2}\right.$, $\alpha / \beta=3$ Gy) was associated with increased risk of developing late vaginal ulcer development, therefore, dose constraint for vagina was set under $145 \mathrm{~Gy}$ [19]. Although no specific dose constraint was set to urethra, urethral catheter was inserted in all cases and it was easy to visualize the urethra, attention was paid not to deliver large dose to urethra. The prescription dose per fraction of HBR-ISBT at our department is $6 \mathrm{~Gy}$. In a whole treatment session, needle insertion was performed once and irradiation was conducted twice daily at 6-hour interval with patients kept in bed overnight throughout the treatment period. Figure 1 shows an example of dose distribution of CT-based image-guided HDR-ISBT.

When calculating the combined dose of EBRT and HDR-ISBT, the equivalent dose in $2 \mathrm{~Gy}$ fractions $\left(\mathrm{EQD}_{2}\right)$ was utilized according to the linear-quadratic dose-effect model $[22,23,24]$. For calculating tumor and OAR doses, $\alpha / \beta$ was assumed as $10 \mathrm{~Gy}$ and $3 \mathrm{~Gy}$, respectively. Dose contribution from CS was not taken into consideration according to Japan Society of Gynecologic Oncology guidelines [15]. The minimum dose covering $90 \%$ of the CTV $\left(C T V D_{90}\right)$ in $\mathrm{EQD}_{2}$ was used as the representative dose of HDR-ISBT.

Patients with histologically proven cervical squamous cell carcinoma, adenocarcinoma, or adenosquamous carcinoma, who were treated by primary radiation therapy including HDR-ISBT with a minimum follow-up period of 24 months were included in this study. Patients with distant metastasis, except para-aortic lymph node (PALN), were excluded from this study. Demographic, disease-specific, treatment details, and outcome data were collected. Survival time was calculated from the start of the radiation therapy until the last follow-up attendance or death from any cause. The local control rate (LC) was calculated from the start of the radiation therapy and histological or apparent radiological evidence of pelvic disease recurrence within the radiation field, which was considered to be an event and was censored at the time of death, non-local relapse, or last follow-up visit. Progression-free survival (PFS) was calculated from the start of the radiation therapy until the last follow-up attendance or any recurrence of the disease. The cumulative overall survival (OS) and PFS 


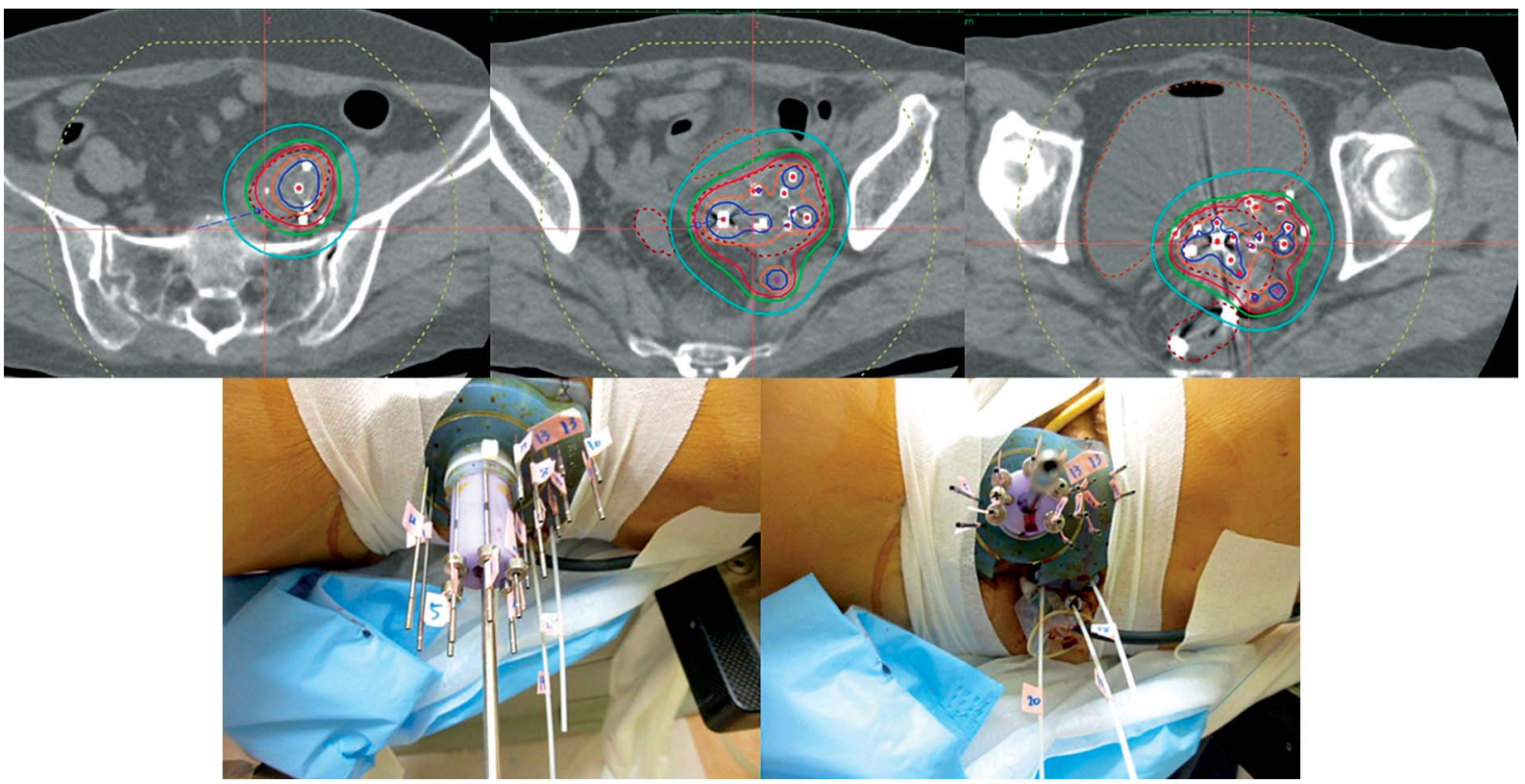

Fig. 1. An example of dose distribution of computed tomography based image-guided high-dose-rate interstitial brachytherapy and pictures of perineal needle insertion through Syed-Neblette template

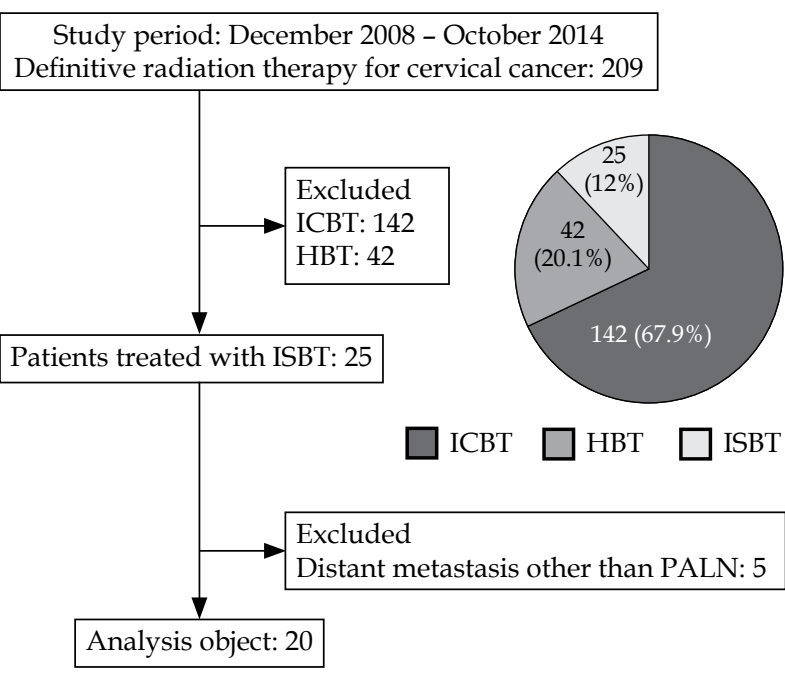

ICBT - intracavitary brachytherapy, HBT - hybrid brachytherapy, ISBT - interstitial brachytherapy, PALN - para-aortic lymph node

Fig. 2. Consolidated Standards of Reporting Trials (CONSORT) flow diagram of uterine cervical patients treated as primary radiation therapy from December 2008 to October 2014 in our hospital

after radiation therapy were calculated by the KaplanMeier method. Univariate survival curve comparisons were performed by the log-rank test. The level of statistically significance was defined as $p<0.05$.

This retrospective study was approved by the Institutional Ethical Review Board of the National Cancer Center Hospital (approval number: 2015-359) and was performed in accordance with the ethical standards laid down in the 1964 Declaration of Helsinki and its later amendments.

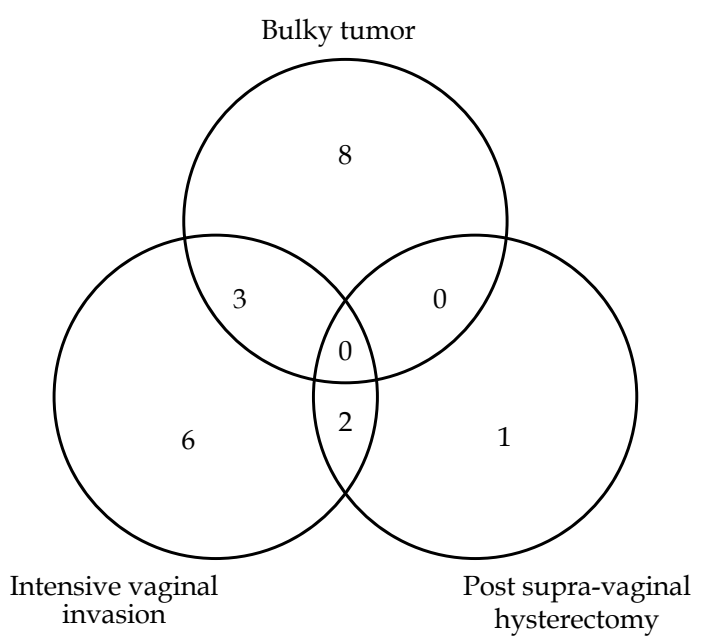

Fig. 3. Venn diagram showing reasons for selecting computed tomography based image-guided high-dose-rate interstitial brachytherapy

\section{Results}

Consolidated Standards of Reporting Trials (CONSORT) flow diagram is shown in Figure 2. From December 2008 to October 2014, 209 consecutive uterine cervical cancer patients were treated with primary radiation therapy. Among them, 142 and 42 patients were treated by ICBT and HBT, respectively. Therefore, these 184 patients were excluded from this study and remaining 25 patients $(12 \%)$ were treated by HDR-ISBT. Patients who had distant metastasis other than PALN were further excluded from the study, therefore, 5 patients were excluded and a total of 20 patients consisted of this analysis. In Figure 3, the reasons for having chosen ISBT are shown. Bulky tumor and intensive lower vaginal invasion were two major reasons for choosing ISBT. 
Table 1. Patients and disease characteristics $(n=20)$

\section{Factor}

\begin{tabular}{|c|c|}
\hline Median age (range), years & $62(42-82)$ \\
\hline \multicolumn{2}{|l|}{ T stage } \\
\hline $3 a$ & 2 \\
\hline $3 b$ & 15 \\
\hline $4 a$ & 3 \\
\hline \multicolumn{2}{|l|}{ N stage } \\
\hline 0 & 7 \\
\hline 1 & 13 \\
\hline \multicolumn{2}{|l|}{ M stage } \\
\hline 0 & 13 \\
\hline 1 & 7 \\
\hline \multicolumn{2}{|l|}{ FIGO stage } \\
\hline IIIA & 2 \\
\hline $\mathrm{IIIB}$ & 10 \\
\hline IVA & 1 \\
\hline IVB & 7 \\
\hline \multicolumn{2}{|l|}{ Histological subtype } \\
\hline Squamous & 16 \\
\hline Adeno & 3 \\
\hline Adenosquamous & 1 \\
\hline Median tumor size at initial presentation $(\mathrm{cm})$ & $7.0(4-14)$ \\
\hline \multicolumn{2}{|l|}{ Hydronephrosis } \\
\hline Yes & 4 \\
\hline No & 16 \\
\hline \multicolumn{2}{|l|}{ Corpus invasion } \\
\hline Yes & 14 \\
\hline No & 6 \\
\hline \multicolumn{2}{|l|}{ Pyometra } \\
\hline Yes & 5 \\
\hline No & 15 \\
\hline $\begin{array}{l}\text { Median hemoglobin value at initial } \\
\text { presentation }(\mathrm{g} / \mathrm{dl})\end{array}$ & $12.2(8.3-13.1)$ \\
\hline
\end{tabular}

Table 1 demonstrates patients and tumor characteristics. All the patients had tumor stage greater than III. As many as 7 patients had radiologically evident PALN metastasis at the time of presentation. Median tumor diameter was as large as $7 \mathrm{~cm}$ (range $4-14 \mathrm{~cm}$ ). Table 2 shows treatment detail. All the patients received higher than 30 Gy of WPRT before delivery of ISBT, and median WPRT dose was $40 \mathrm{~Gy}$. Median fractions of ISBT was 4 times (range 3-6). Median size of HR-CTV measured by CT taken after needle insertion was $115.5 \mathrm{ml}$ (range 6.6$175.6 \mathrm{ml}$ ), and median HR-CTV $\mathrm{D}_{90}$ in $\mathrm{EQD}_{2}$ was $74.2 \mathrm{~Gy}$ (60.4-88.5 Gy). Because radioactivity of iridium source
Table 2. Treatment details $(n=20)$

Factor

\begin{tabular}{|c|c|}
\hline $\begin{array}{l}\text { Median dose prescribed to whole } \\
\text { pelvice (Gy) }\end{array}$ & $40(30-50.4)$ \\
\hline HDR-ISBT fractions $(n)$ & $4(3-6)$ \\
\hline Median number of needles used in ISBT & $18.5(10-33)$ \\
\hline $\begin{array}{l}\text { Percentage of dose delivered from } \\
\text { interstitial component* }(\%)\end{array}$ & $68.2(17.7-95.2)$ \\
\hline Median volume of HR-CTV (ml) & $115.5(6.6-175.6)$ \\
\hline Median dose of HR-CTV $D_{90}$ in EQD ${ }_{2}$ (Gy) & $74.2(60.4-88.5)$ \\
\hline Median dose of bladder $\mathrm{D}_{2 c \mathrm{c}}$ in $\mathrm{EQD}_{2}$ (Gy) & $71.0(48.4-84.0)$ \\
\hline Median dose of rectum $\mathrm{D}_{2 c c}$ in $\mathrm{EQD}_{2}$ (Gy) & $67.5(52.3-76.4)$ \\
\hline \multicolumn{2}{|l|}{ Concurrent chemotherapy } \\
\hline Yes & 17 \\
\hline No & 3 \\
\hline Median overall treatmet time (weeks) & $6.4(5.3-8.3)$ \\
\hline
\end{tabular}

${ }^{*}$ Ratio of dwell time of all intersititial needles divided by total treatment time. Patients who received supra-vaginal hysterectomy for uterine myoma $(n=3)$ were excluded from the analysis because no tandem was inserted in those patients.

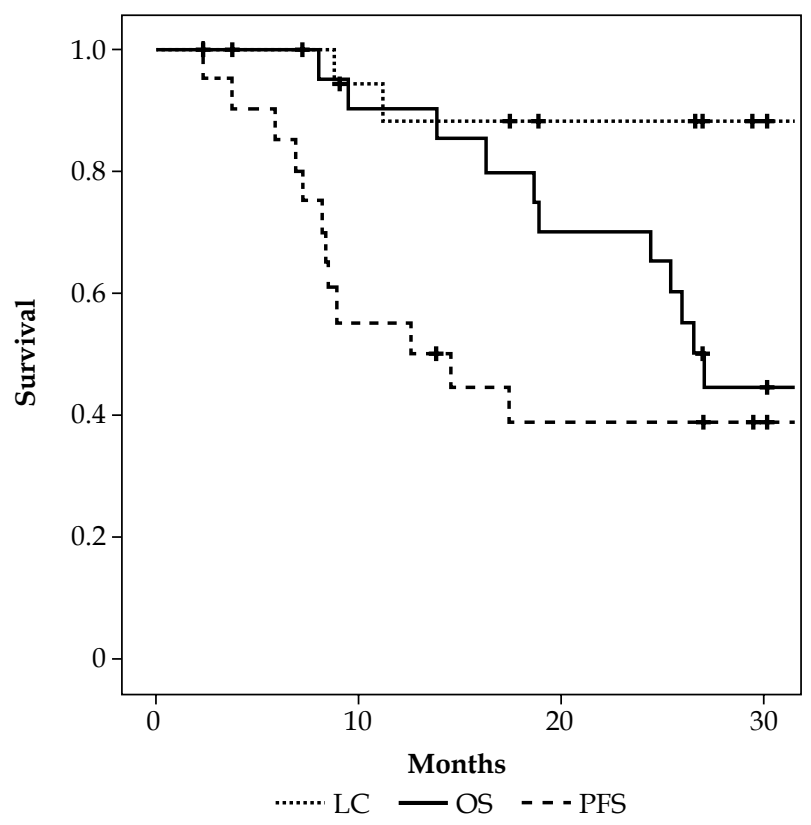

Fig. 4. Kaplan-Meier survival curves for local control (LC), progression-free survival (PFS), and overall survival (OS) of uterine cervical cancer patients without distant metastasis other than para-aortic lymph nodes treated by computed tomography based image-guided high-dose-rate interstitial brachytherapy

varies from time to time, percentage of interstitial component was calculated by dividing sum of dwell time of all interstitial needles with total treatment time. Patients who received supra-vaginal hysterectomy for uterine myoma $(n=3)$ were excluded from this calculation, because no tandem was inserted in those patients. Median percentage of dose delivered from interstitial component was $68.2 \%$ (range $17.7-95.2 \%$ ). 
Median follow-up period for patients still alive at last contact was 32.0 months (range 27.0-67.2 months). Threeyear OS, PFS, and LC were $44.4 \%, 38.9 \%$, and $87.8 \%$, respectively (Figure 4). At the time of analysis, seven patients were alive without disease relapse, while eleven patients died because of progression of cervical cancer, and one due to other reasons without any evidence of disease progression. Figure 5 demonstrates patterns of first relapse. Major site of relapse was distant metastasis and only two local recurrences were found. Clinical factors were compared between patients with or without disease progression or local relapse by univariate analysis (Tables 3 and 4). Patients with local relapse had significantly higher ratio of hydronephrosis and lower hemoglobin value at initial presentation ( $p=0.032$ and $p=0.032$, respectively).

\section{Treatment related toxicities}

One patient experienced grade 3 rectal bleeding requiring hospitalization and blood infusion, although the rectum $\mathrm{D}_{2 \mathrm{cc}}$ of this patient was $61.5 \mathrm{~Gy}$. This patient underwent intra-arterial infusion of chemotherapy despite of no evidence of disease progression at the primary hospital after receiving HDR-ISBT. Therefore, it can be assumed that such intra-arterial treatment, which has influence on circulation of normal tissue, would have
Pelvic recurrence

(local recurrence: 2 , pelvic lymph node: 1 )

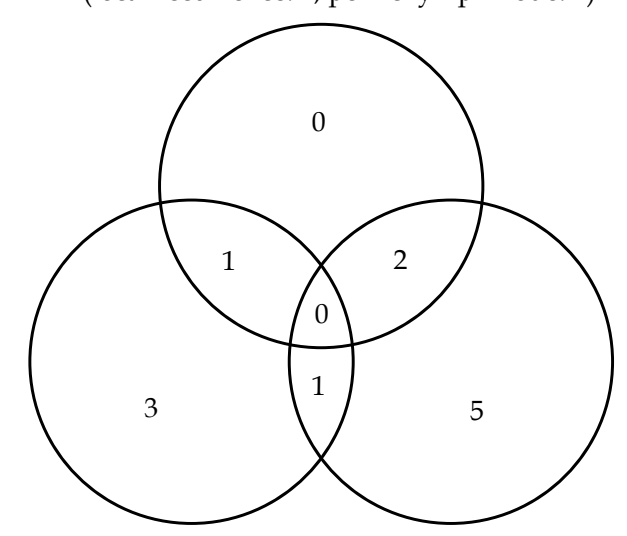

Para-aortic lymph node

Distant metastasis

Fig. 5. Venn diagram showing patterns of first relapse after computed tomography based image-guided high-dose-rate interstitial brachytherapy

altered surrounding environment. Four patients experienced grade 2 rectal bleeding, one grade 2 cystitis, and two grade 2 vaginal ulcer.

Table 3. Potential predictors influencing disease progression

\begin{tabular}{|c|c|c|c|}
\hline & Without disease progression $(n=8)$ & With disease progression $(n=12)$ & $p$ value \\
\hline \multicolumn{4}{|l|}{ Histological subtype } \\
\hline Squamous & 7 & 8 & 0.307 \\
\hline Non-squamous & 1 & 4 & \\
\hline \multicolumn{4}{|l|}{ M stage } \\
\hline MO & 7 & 6 & 0.106 \\
\hline MA & 1 & 6 & \\
\hline Initial tumor size $(\mathrm{cm})$ & $7.7(4-14)$ & $7.9(6.4-11.4)$ & 0.821 \\
\hline \multicolumn{4}{|l|}{ Hydronephrosis } \\
\hline Yes & 1 & 3 & 0.465 \\
\hline No & 7 & 9 & \\
\hline \multicolumn{4}{|l|}{ Corpus invasion } \\
\hline Yes & 5 & 9 & 0.455 \\
\hline No & 3 & 3 & \\
\hline \multicolumn{4}{|l|}{ Pyometra } \\
\hline Yes & 2 & 3 & 0.693 \\
\hline No & 6 & 9 & \\
\hline $\begin{array}{l}\text { Hemoglobin value at initial } \\
\text { presentation }(\mathrm{g} / \mathrm{dl})\end{array}$ & $12.4(11.6-13.1)$ & $11.5(8.3-12.8)$ & 0.085 \\
\hline \multicolumn{4}{|l|}{ Concurrent chemotherapy } \\
\hline Yes & 8 & 9 & 0.193 \\
\hline No & 0 & 3 & \\
\hline HR-CTV $D_{90}$ in EQD 2 (Gy) & $74.3(67.3-80.8)$ & $72.9(60.4-88.5)$ & 0.687 \\
\hline HR-CTV (ml) & $119.2(6.6-175.6)$ & $115.5(28.2-167.9)$ & 0.336 \\
\hline
\end{tabular}


Table 4. Potential predictors influencing local relapse

\begin{tabular}{|c|c|c|c|}
\hline & Without local relapse $(n=18)$ & With local relapse $(n=2)$ & $p$ value \\
\hline \multicolumn{4}{|l|}{ Histological subtype } \\
\hline Squamous & 14 & 1 & 0.447 \\
\hline Non-squamous & 4 & 1 & \\
\hline \multicolumn{4}{|l|}{ T stage } \\
\hline $\mathrm{T3}$ & 15 & 2 & 0.716 \\
\hline $\mathrm{T} 4$ & 3 & 0 & \\
\hline Initial tumor size $(\mathrm{cm})$ & $7.8(4-14)$ & $7.9(6.4-11.4)$ & 0.963 \\
\hline \multicolumn{4}{|l|}{ Hydronephrosis } \\
\hline Yes & 2 & 2 & $0.032^{\star}$ \\
\hline No & 16 & 0 & \\
\hline \multicolumn{4}{|l|}{ Corpus invasion } \\
\hline Yes & 12 & 2 & 0.479 \\
\hline No & 6 & 0 & \\
\hline \multicolumn{4}{|l|}{ Pyometra } \\
\hline Yes & 4 & 1 & 0.447 \\
\hline No & 14 & 1 & \\
\hline $\begin{array}{l}\text { Hemoglobin value at initial } \\
\text { presentation }(\mathrm{g} / \mathrm{dl})\end{array}$ & $12.4(11.6-13.1)$ & $11.5(8.3-12.8)$ & $0.032^{*}$ \\
\hline \multicolumn{4}{|l|}{ Concurrent chemotherapy } \\
\hline Yes & 15 & 2 & 0.716 \\
\hline No & 3 & 0 & \\
\hline HR-CTV $D_{90}$ in EQD $(G y)$ & $72.8(60.4-88.5)$ & $80.1(75.1-85.1)$ & 0.191 \\
\hline HR-CTV (ml) & $115.5(6.6-175.6)$ & $131.55(105-139.2)$ & 0.631 \\
\hline
\end{tabular}

\section{Discussion}

Interstitial brachytherapy with transperineal approach in locally advanced cervical cancer is a well-established method for decades and there are numerous studies on ISBT; the vast majority of them are from $2 \mathrm{D}$ era $[25,26$, $27,28]$. However, there is also an increasing number of studies based on 3D image-based brachytherapy $[19,20,29,30,31]$. Most of the reports of ISBT focused only on patients treated with ISBT, but current study overlooked all cervical cancer patients in a single institution who were treated by brachytherapy including ICBT, HBT, and ISBT, and reported the proportion of patients treated by ISBT. It has been shown from several reports that most of the tumors can be treated with ICBT or HBT. Nevertheless, there still exists a small group of patients whose tumors require ISBT to achieve better local control at a cost of its invasiveness. In current study, $12 \%$ of uterine cervical patients who were treated with primary radiation therapy received HDR-ISBT. Compared with recently published retro EMBRACE study, a multicenter cohort study that reported clinical outcome of image-guided brachytherapy in locally advanced cervical cancer [32], this number is relatively high. In the retro EMBRACE study, median tumor width at diagnosis was $5 \mathrm{~cm}$ and 168 out of 731 patients
$(23.0 \%)$ were treated with HBT. One of the reasons why so many patients were treated with ISBT in our institution was that, because there are only few institutions in our country capable to perform ISBT, and such patients who could not be well-treated with conventional ICBT were referred to our institution. Another reason was incorporation of CS in our treatment schedule. Compared to treatment schedule without using CS, brachytherapy starts earlier before any considerable tumor shrinkage from proceeding pelvic EBRT is allowed.

Local control after HDR-ISBT was favorable despite having several adverse clinical factors such as large tumor size or advanced stage (Table 1). However, in current study, distant metastases were frequently observed, suggesting that microscopic distant metastasis would supposedly have existed at initial presentation. Currently, OUTBACK trial is under way [33], which tests the superiority of adjuvant chemotherapy after cCRT over cCRT alone for locally advanced uterine cervical cancer patients. If the result of this trial will be positive, it is possible that adjuvant chemotherapy would be beneficial for patients whose primary tumor require HDR-ISBT.

Median HR-CTV $\mathrm{D}_{90}$ in EQD2 was 74.2 Gy and it was lower than $87 \mathrm{~Gy}$, which was recommended prescribed dose for HR-CTV proposed by Dimopoulos et al. and 
ABS guidelines [1,2,7]. However, 74.2 Gy was calculated by simply adding the dose from WPRT and ISBT, and dose contribution from CS, which is utilized to minimize dose delivered to the rectum or the bladder neck, was not taken into account according to Japan Society of Gynecologic Oncology guideline [15]. Recently, Tamaki et al. calculated total dose delivered to HR-CTV including dose from CS using deformable image registration technique, and reported that actual dose delivered to HR-CTV was higher than simple summation of WPRT and ISBT [34]. Although, deformable image registration technique has its own limitation, the anatomical relationship would change dramatically with the applicators in place compared with the EBRT situation. Additionally, in our treatment strategy, timing of delivering brachytherapy is earlier than that of treatment schedule without using CS. In the treatment schedule, in which no CS is applied, brachytherapy is delivered after whole EBRT. In contrast, in a treatment schedule with CS, brachytherapy is started after initiation of CS. Therefore, overall treatment time is much shorter in treatment schedule with CS than that of without CS. Toita et al. showed the efficacy of treatment schedule with CS in a series of multicenter prospective clinical trials [35,36], and our group demonstrated favorable results with schedule with CS in CT based 3D imageguided brachytherapy [16].

Hybrid brachytherapy is much easier to perform compared with ISBT because it only requires a few interstitial needles and could be performed in outpatient setting [11]. In contrast, ISBT demands time and labor, and forces the patients to lay on the bed overnight during treatment period. Consequently, it is very hard to make ISBT equally accessible nationwide. Therefore, it is important to build a national system, in which patients who require ISBT could be referred to high-volume centers efficiently. Current matter in question is that there exist no concrete guidelines for selecting ICBT, HBT, or ISBT for locally advanced uterine cervical cancer. Yoshida et al. recently published simulation analysis and they proposed tumors that was larger than $5 \times 4 \times 4 \mathrm{~cm}$ were treated by ISBT [37]. Because they used simple rectangular imaginary tumor for their analysis, the results should be interpreted with caution. If more applicable guideline will be established, patient selection will become easier.

There were several limitations in our study that was a retrospective study from a single institution with a quite limited number of patients. Because of the limited number of patients, any analytical results derived from this study should be interpreted with a caution. Therefore, the results of this study should not be generalized. A prospective trial is warranted to validate the superiority of ISBT over other types of brachytherapy methods in locally advanced uterine cervical cancer patients.

\section{Conclusions}

Feasibility and favorable local control of interstitial brachytherapy for locally advanced cervical cancer was demonstrated through a single institutional experience with a small number of patients.

\section{Acknowledgments}

This study was partially supported by the Japan Agency for Medical Research and Development, AMED, the National Cancer Center Research and Development Fund (26-A-18 and 26-A-28), and JSPS KAKENHI Grant Number 15K19836.

\section{Disclosure}

Authors report no conflict of interest.

\section{References}

1. Viswanathan AN, Thomadsen B. American Brachytherapy Society Cervical Cancer Recommendations Committee; American Brachytherapy Society. American Brachytherapy Society consensus guidelines for locally advanced carcinoma of the cervix. Part I: general principles. Brachytherapy 2012; 11: 33-46.

2. Viswanathan AN, Beriwal S, De Los Santos JF et al. American Brachytherapy Society consensus guidelines for locally advanced carcinoma of the cervix. Part II: high-dose-rate brachytherapy. Brachytherapy 2012; 11: 47-52.

3. Bethesda M. ICRU report 38. Dose and volume specification for reporting intracavitary therapy in gynecology. ICRU 1985; 38: 1-20.

4. Tod M, Meredith WJ. Treatment of cancer of the cervix uteri, a revised Manchester method. Br J Radiol 1953; 26: 252-257.

5. Haie-Meder C, Pötter R, Van Limbergen E et al. Recommendations from Gynaecological (GYN) GEC-ESTRO Working Group (I): concepts and terms in 3D image based 3D treatment planning in cervix cancer brachytherapy with emphasis on MRI assessment of GTV and CTV. Radiother Oncol 2005; 74: 235-245.

6. Pötter R, Haie-Meder C, Van Limbergen E et al. Recommendations from gynaecological (GYN) GEC ESTRO working group (II): concepts and terms in 3D imagebased treatment planning in cervix cancer brachytherapy-3D dose volume parameters and aspects of 3D image-based anatomy, radiation physics, radiobiology. Radiother Oncol 2006; 78: 67-77.

7. Dimopoulos JC, Lang S, Kirisits C et al. Dose-volume histogram parameters and local tumor control in magnetic resonance image-guided cervical cancer brachytherapy. Int $J$ Radiat Oncol Biol Phys 2009; 75: 56-63.

8. Nag S, Cardenes H, Chang S et al. Proposed guidelines for image-based intracavitary brachytherapy for cervical carcinoma: report from Image-Guided Brachytherapy Working Group. Int J Radiat Oncol Biol Phys 2004; 60: 1160-1172.

9. Dimopoulos JC, Kirisits C, Petric P et al. The Vienna applicator for combined intracavitary and interstitial brachytherapy of cervical cancer: clinical feasibility and preliminary results. Int J Radiat Oncol Biol Phys 2006; 66: 83-90.

10. Jürgenliemk-Schulz IM, Tersteeg RJ, Roesink JM et al. MRIguided treatment-planning optimization in intracavitary or combined intracavitary/interstitial PDR brachytherapy using tandem ovoid applicators in locally advanced cervical cancer. Radiother Oncol 2009; 93: 322-330.

11. Tan PW, Koh VY, Tang JI. Outpatient combined intracavitary and interstitial cervical brachytherapy: barriers and solutions to implementation of a successful programme - a single institutional experience. J Contemp Brachytherapy 2015; 7: 259-263.

12. Bailleux C, Falk AT, Chand-Fouche ME et al. Concomitant cervical and transperineal parametrial high-dose-rate brachytherapy boost for locally advanced cervical cancer. J Contemp Brachytherapy 2016; 8: 23-31.

13. Kuroda Y, Murakami N, Morota M et al. Impact of concurrent chemotherapy on definitive radiotherapy for women with FIGO IIIb cervical cancer. J Radiat Res 2012; 53: 588-593. 
14. Toita T, Ohno T, Kaneyasu Y et al. A consensus-based guideline defining the clinical target volume for pelvic lymph nodes in external beam radiotherapy for uterine cervical cancer. Jpn J Clin Oncol 2010; 40: 456-463.

15. Ebina Y, Yaegashi N, Katabuchi H et al. Japan Society of Gynecologic Oncology guidelines 2011 for the treatment of uterine cervical cancer. Int J Clin Oncol 2015; 20: 240-248.

16. Murakami N, Kasamatsu T, Wakita A et al. CT based threedimensional dose-volume evaluations for high-dose rate intracavitary brachytherapy for cervical cancer. BMC Cancer 2014; 14: 447-454.

17. Yoshio K, Murakami N, Morota M et al. Inverse planning for combination of intracavitary and interstitial brachytherapy for locally advanced cervical cancer. J Radiat Res 2013; 54: 1146-1152.

18. Itami J, Hara R, Kozuka T et al. Transperineal high-dose-rate interstitial brachytherapy in the management of gynecologic malignancies. Strahlenther Onkol 2003; 179: 737-741.

19. Murakami N, Kasamatsu T, Sumi M et al. Vaginal tolerance of CT based image-guided high-dose rate interstitial brachytherapy for gynecological malignancies. Radiat Oncol 2014; 9: 31-38.

20. Murakami N, Kato T, Miyamoto Y et al. Salvage high-doserate interstitial brachytherapy for pelvic recurrent cervical carcinoma after hysterectomy. Anticancer Res 2016; 36: 2413 2421.

21. Rao PB, Ghosh S. Routine use of ultrasound guided tandem placement in intracavitary brachytherapy for the treatment of cervical cancer - a South Indian institutional experience. J Contemp Brachytherapy 2015; 7: 352-356.

22. Dale RG. The application of the linear-quadratic dose-effect equation to fractionated and protracted radiotherapy. Br J Radiol 1985; 58: 515-528.

23. Bentzen SM, Dörr W, Gahbauer R et al. Bioeffect modeling and equieffective dose concepts in radiation oncology - terminology, quantities and units. Radiother Oncol 2012; 105: 266-268.

24. Fowler JF. Sensitivity analysis of parameters in linear-quadratic radiobiologic modeling. Int J Radiat Oncol Biol Phys 2009; 73: 1532-1537.

25. Martinez A, Cox RS, Edmundson GK. A multiple-site perineal applicator (MUPIT) for treatment of prostate, anorectal, and gynecologic malignancies. Int J Radiat Oncol Biol Phys 1984; 10: 297-305.

26. Syed AM, Puthawala AA, Abdelaziz NN et al. Long-term results of low-dose-rate interstitial-intracavitary brachytherapy in the treatment of carcinoma of the cervix. Int J Radiat Oncol Biol Phys 2002; 54: 67-78.

27. Nag S, Martinez-Monge R, Selman AE et al. Interstitial brachytherapy in the management of primary carcinoma of the cervix and vagina. Gynecol Oncol 1998; 70: 27-32.

28. Demanes DJ, Rodriguez RR, Bendre DD et al. High dose rate transperineal interstitial brachytherapy for cervical cancer: high pelvic control and low complication rated. Int J Radiat Oncol Biol Phys 1999; 45: 105-112.

29. Yoshida K, Yamazaki H, Takenaka T et al. A dose-volume analysis of magnetic reasonance imaging-aided high-doserate image-based interstitial brachytherapy for uterine cervical cancer. Int J Radiat Oncol Biol Phys 2010; 77: 765-772.

30. Yoshida K, Yamazaki H, Takenaka T et al. Preliminary results of MRI-assisted high-dose-rate interstitial brachytherapy for uterine cervical cancer. Brachytherapy 2015; 14: 1-8.

31. Lee JI, Viswanathan AN. Predictors of toxicity after imageguided high-dose-rate interstitial brachytherapy for gynecologic cancer. Int J Radiat Oncol Biol Phys 2012; 84: 1192-1197.

32. Sturdza A, Pötter R, Fokdal LU et al. Image guided brachytherapy in locally advanced cervical cancer: improved pelvic control and survival in RetroEMBRACE, a multicenter cohort study. Radiother Oncol 2016; 120: 428-433.

33. Mileshkin L, Narayan K, King M et al. A phase III trial of adjuvant chemotherapy as primary treatment for locally advanced cervical cancer compared to chemoradiation alone: The OUTBACK Trial (ANZGOG 0902/GOG-0274/RTOG 1174).

34. Tamaki T, Ohno T, Noda $S$ et al. Filling the gap in central shielding: three-dimensional analysis of the EQD2 dose in radiotherapy for cervical cancer with the central shielding technique. J Radiat Res 2015; 56: 804-810.

35. Toita T, Kitagawa R, Hamano T et al. Phase II study of concurrent chemoradiotherapy with high-dose-rate intracavitary brachytherapy in patients with locally advanced uterine cervical cancer: efficacy and toxicity of a low cumulative radiation dose schedule. Gynecol Oncol 2012; 126: 211-216.

36. Toita T, Kato $S$, Niibe $Y$ et al. Prospective multi-institutional study of definitive radiotherapy with high-dose-rate intracavitary brachytherapy in patients with nonbulky $(<4-\mathrm{cm})$ stage I and II uterine cervical cancer (JAROG0401/JROSG04-2). Int J Radiat Oncol Biol Phys 2012; 82: e49-56.

37. Yoshida K, Yamazaki H, Kotsuma T et al. Simulation analysis of optimized brachytherapy for uterine cervical cancer: Can we select the best brachytherapy modality depending on tumor size? Brachytherapy 2016; 15: 57-64. 